\title{
Finite Difference Method for Numerical Solution of Two Point Boundary Value Problems with Non-uniform Mesh and Internal Boundary Condition
}

\author{
P. K. Pandey \\ Department of Mathematics, Dyal Singh College (Univ. of Delhi), Lodhi Road, New Delhi-110003, India \\ pramod_10p@hotmail.com
}

\begin{abstract}
In this article, we have presented a variable step finite difference method for solving second order boundary value problems in ordinary differential equations with an internal boundary condition. We have discussed the convergence and established at least cubic order of accuracy of the proposed method. The proposed method tested on several model problems for the numerical solution. The numerical results obtained for these model problems with known / constructed exact solution confirm the theoretical conclusions of the proposed method. The computational results obtained for these model problems suggest that method is efficient and accurate.
\end{abstract}

Keywords: Boundary Value Problem, Convergence of the Method, Cubic Order, Finite Difference Method, Variable Step.

\section{Introduction}

Recently, there has been a great deal of interest in developing methods for the numerical solution of two-point boundary value problems. These methods deal without an internal boundary condition and it is our purpose here to develop finite-difference method to deal with an internal boundary condition.In this article we considered a finite difference method for the numerical solution of the boundary value problems of the form

$$
u^{\prime \prime}(x)=f(x, u), \quad a \leq x<\frac{a+b}{2}, \quad \frac{a+b}{2}<x<b
$$

subject to the boundary conditions

$$
u\left(\frac{a+b}{2}\right)=\alpha \quad \text { and } \quad u(b)=\beta
$$

or

$$
u(a)=\alpha \quad \text { and } \quad u\left(\frac{a+b}{2}\right)=\beta,
$$

where $\alpha$ and $\beta$ are real constants. We assume that $f$ is continuous function in $[a, b] \times \Re$.

In these problems we are unable to integrate from one side of the domain to the other. So it will be difficult to solve these problems numerically by using Numerov method. It is possible to solve the problem away from the internal point at which value is prescribed if we have smooth derivative and solution in the neighborhood of this internal point. To solve problem (1) with the prescribed either boundary conditions, a fitting point method can be applied [8]. 
Ordinary differential equations are used to understand different kind of physical problems in nature. To understand how nature works, we need to solve these differential equations using some analytical methods which satisfy the certain conditions. In most cases, it is impossible to solve these modeled problems under realistic conditions analytically. So we prefer other mathematical techniques to solve these problems. One such technique is numerical approximation / solution and a literature regarding the numerical solution of the two-point boundary value problem is given in 6, 5, 7, 1. The existence and uniqueness of the solution for the problem (1) is assumed. The specific assumption on $f(x, u)$ to ensure existence and uniqueness will not be considered in this article, however I may refer literature in [5, 2, 3]. Also we have assumed that problem (1) is well posed with continuous derivatives and that the solution depends differentially on the boundary and internal conditions. Some literary work on internal boundary condition can be found in [8] but to the best of our knowledge, method similar to the proposed method for the numerical solution of the specific problem (1), have not been discussed in the literature to date.

In this article we shall develop a Numerov Type finite difference method for solving problem (1) numerically. The order and accuracy of the proposed method under certain conditions is four. A numerical experiment performed to demonstrate the effectiveness of the method.

We have presented our work in this article as follows. In the next section we will discuss the finite difference method and in Section 3 we will derive our propose method. In Section 4, we have discussed convergence under appropriate condition. The applications of the proposed method to the model problems and illustrative numerical results have been produced to show the efficiency in Section 5. Discussion and conclusion on the performance of the method are presented in Section 6.

\section{The Finite Difference Method}

We define $N-1$ odd and finite numbers of nodal points in $[a, b]$, the domain of solution of the problem (1) i.e. $a \leq x_{0}<x_{1}<x_{2}<\ldots .<x_{N}=b$ using nonuniform step length $h_{i}$ such that $x_{i+1}=x_{i}+h_{i+1}, \quad i=0,1, . . N$ and $x_{\frac{N}{2}}=\frac{b-a}{2}$. We wish to determine the numerical approximation of the theoretical solution $u(x)$ of the problem $(1)$ at the nodal point $x_{i}, \quad i=0,1,2, . ., \frac{N}{2}-1, \frac{N}{2}+1, \ldots, N-1$. To simplify we denote the numerical approximation of $u(x)$ at the node $x=x_{i}$ as $u_{i}$. Let us denote $f_{i}$ as the approximation of the theoretical value of the source function $f(x, u(x))$ at node $x=x_{i}, \quad i=0,1,2, \ldots ., N$. Thus we can define other notations used in this article i.e. $f_{i \pm 1}, \quad$ and $u_{i \pm 1}$, in the similar way. Applying these defined notations, the differential equation (1) at the mesh point $x=x_{i}$ may be written as,

$$
u_{i}^{\prime \prime}=f\left(x_{i}, u_{i}\right)
$$

Hence, in order to discretize problem (1) at the mesh points we approximate the differential equation (2) by a difference formula of Numerov Type,

$$
a_{2 i} y_{i+1}+a_{1 i} y_{i}+a_{0 i} y_{i-1}=C_{2 i} f_{i+1}+C_{1 i} f_{i}+C_{0 i} f_{i-1}, \quad i=1,2, \ldots \ldots, N-1,
$$

where the coefficients $a_{0 i}, a_{1 i}, a_{2 i}, C_{0 i}, C_{1 i}$, and $C_{2 i}$ are not constant and function of $r_{i}=\frac{h_{i+1}}{h_{i}}$. We can obtain these coefficients in a similar way as on an equidistant mesh by method of Taylor series expansion and undetermine coefficients. Let these coefficients

$$
\begin{aligned}
& a_{2 i}=1, \quad a_{1 i}=-\left(1+r_{i}\right), \quad a_{0 i}=r_{i}, \quad C_{0 i}=\frac{h_{i}^{2}}{12} r_{i}\left(1+r_{i}-r_{i}^{2}\right), \\
& C_{1 i}=\frac{h_{i}^{2}}{12}\left(1+r_{i}\right)\left(1+3 r_{i}+r_{i}^{2}\right), C_{2 i}=\frac{h_{i}^{2}}{12}\left(-1+r_{i}+r_{i}^{2}\right)
\end{aligned}
$$


Thus we propose our difference method for the numerical solution of problem (1),

$$
\begin{array}{r}
r_{i} u_{i-1}-\left(1+r_{i}\right) u_{i}+u_{i+1}=C_{0 i} f_{i-1}+C_{1 i} f_{i}+C_{2 i} f_{i+1}, \quad 1 \leq i \leq \frac{N}{2}-2 \\
r_{i} u_{i-1}-\left(1+r_{i}\right) u_{i}=-u_{i+1}+C_{0 i} f_{i-1}+C_{1 i} f_{i}+C_{2 i} f_{i+1}, \quad i=\frac{N}{2}-1 \\
r_{i} u_{i-1}+u_{i+1}=\left(1+r_{i}\right) u_{i}+C_{0 i} f_{i-1}+C_{1 i} f_{i}+C_{2 i} f_{i+1}, \quad i=\frac{N}{2} \\
-\left(1+r_{i}\right) u_{i}+u_{i+1}=-r_{i} u_{i-1}+C_{0 i} f_{i-1}+C_{1 i} f_{i}+C_{2 i} f_{i+1}, \quad i=\frac{N}{2}+1 \\
r_{i} u_{i-1}-\left(1+r_{i}\right) u_{i}+u_{i+1}=C_{0 i} f_{i-1}+C_{1 i} f_{i}+C_{2 i} f_{i+1}, \quad \frac{N}{2}+2 \leq i \leq N-2 \\
r_{i} u_{i-1}-\left(1+r_{i}\right) u_{i}=-u_{i+1}+C_{0 i} f_{i-1}+C_{1 i} f_{i}+C_{2 i} f_{i+1}, \quad i=N-1 .
\end{array}
$$

which is a $N-1 \times N-1$ system of linear/ nonlinear equations depending on forcing function $f(x, u)$. If forcing function is linear then it will be system of linear equations otherwise system of nonlinear equations. We have applied Gauss Seidel method in case of solving system of linear equations and Newton Raphson method in case of solving system of nonlinear equations.

\section{Local Truncation Error}

The local truncation error in the difference method (5) using the exact arithmetic may be calculated by using Taylor series expansion at the nodal points $x=x_{i-1}, i=1,2, \frac{N}{2}, \frac{N}{2}+2, \ldots, N$. Thus the truncation error $T_{i-1}$ in method (5) may be written as :

$$
T_{i-1}=\left\{\begin{array}{lr}
\frac{h_{i}^{5} r_{i}\left(r_{i}+1\right)\left(2-3 r_{i}+7 r_{i}^{2}-12 r_{i}^{3}\right)}{360} u_{i-1}^{(5)}, & 1 \leq i \leq \frac{N}{2} \\
\frac{-h_{i}^{5}\left(r_{i}-1\right)\left(8+5 r_{i}+5 r_{i}^{2}\right)}{360} u_{i-1}^{(5)}, & \frac{N}{2}+2 \leq i \leq N .
\end{array}\right.
$$

Thus we have obtained a truncation error at each node of $O\left(h_{i}^{5}\right)$.

\section{Convergence of the Difference Method}

Let $U_{i}=u\left(x_{i}, u\left(x_{i}\right)\right)$ and $u_{i}$ be respectively an exact solution and approximate solution of the problem. Let define the forcing function in problem (1),

$$
\begin{gathered}
f_{i}=f\left(x_{i}, u_{i}\right) \\
F_{i}=f\left(x_{i}, U_{i}\right) .
\end{gathered}
$$

Apply method of linearization technique on forcing functions, we will obtain the following,

$$
\begin{array}{r}
F_{i}=f\left(x_{i}, U_{i}\right)=f\left(x_{i}, u_{i}\right)+\left(U_{i}-u_{i}\right) G_{i} \\
F_{i \pm 1}=f\left(x_{i \pm 1}, U_{i \pm 1}\right)=f\left(x_{i \pm 1}, u_{i \pm 1}\right)+\left(U_{i \pm 1}-u_{i \pm 1}\right) G_{i \pm 1}
\end{array}
$$

where $G_{i}=\left(\frac{\partial f}{\partial u}\right)_{i}$ and $G_{i \pm 1}=\left(\frac{\partial f}{\partial u}\right)_{i \pm 1}$. Consider the difference method (5),

$$
r_{i} u_{i-1}-\left(1+r_{i}\right) u_{i}+u_{i+1}=C_{0 i} f_{i-1}+C_{1 i} f_{i}+C_{2 i} f_{i+1}
$$

where $C_{0 i}=\frac{h_{i}^{2}}{12} r_{i}\left(1+r_{i}-r_{i}^{2}\right), C_{1 i}=\frac{h_{i}^{2}}{12}\left(1+r_{i}\right)\left(1+3 r_{i}+r_{i}^{2}\right)$ and $C_{2 i}=\frac{h_{i}^{2}}{12}\left(-1+r_{i}+r_{i}^{2}\right)$.

$$
r_{i} U_{i-1}-\left(1+r_{i}\right) U_{i}+U_{i+1}=C_{0 i} F_{i-1}+C_{1 i} F_{i}+C_{2 i} F_{i+1}+T_{i-1}
$$

where $T_{i-1}$ is the truncation error at node $x_{i-1}$.

Let us define $\epsilon_{i}=u_{i}-U_{i}$ and $\epsilon_{i \pm 1}=u_{i \pm 1}-U_{i \pm 1}$. Using these definitions and subtracting (9) from (8), we have obtained,

$$
\begin{gathered}
r_{i} \epsilon_{i-1}-\left(1+r_{i}\right) \epsilon_{i}+\epsilon_{i+1}=C_{0 i}\left(f_{i-1}-F_{i-1}\right)+C_{1 i}\left(f_{i}-F_{i}\right)+C_{2 i}\left(f_{i+1}+F_{i+1}\right)-T_{i-1} \\
\left(r_{i}+C_{0 i} G_{i-1}\right) \epsilon_{i-1}-\left(1+r_{i}+C_{1 i} G_{i}\right) \epsilon_{i}+\left(1+C_{2 i} G_{i+1}\right) \epsilon_{i+1}+T_{i-1}=0
\end{gathered}
$$


Let us write (10) in matrix form

$$
\mathbf{D} \epsilon+\mathbf{T}=\mathbf{0},
$$

where matrix $\mathbf{D}=\left(d_{l m}\right)_{l, m=1}^{N-1}$ be portioned in the following manner,

$$
\mathbf{D}=\left(\begin{array}{ll}
\mathbf{D}_{11} & \mathbf{D}_{12} \\
\mathbf{D}_{21} & \mathbf{D}_{22}
\end{array}\right)_{N-1 \times N-1}
$$

where $\mathbf{D}_{l m}, l<N-1$ are matrices and

$$
\mathbf{D}_{11}=\left(\begin{array}{cccc}
r_{1}+C_{01} G_{0} & -\left(1+r_{1}+C_{11} G_{1}\right) & 1+C_{22} G_{2} & 0 \\
& r_{2}+C_{02} G_{1} & -\left(1+r_{2}+C_{12} G_{2}\right) & 1+C_{23} G_{3} \\
. . & . . & . . \\
. . & r_{\frac{N}{2}-1}+C_{0\left(\frac{N}{2}-1\right)} G_{\frac{N}{2}-2} & -\left(1+r_{\frac{N}{2}-1}+C_{1 \frac{N}{2}} G_{\frac{N}{2}}\right. \\
0 & & & r_{\frac{N}{2}}+C_{0 \frac{N}{2}} G_{\frac{N}{2}-1}
\end{array}\right)
$$

$\frac{N}{2} \times \frac{N}{2}$ matrix with an upper bandwidth of 2,

$$
\mathbf{D}_{12}=\left(\begin{array}{ccc}
0 & . . & 0 \\
. . & . . & . . \\
. . & . . & . . \\
1+C_{2 \frac{N}{2}} G_{\frac{N}{2}+1} & 0 & 0
\end{array}\right) \quad \mathbf{D}_{21}=\left(\begin{array}{ccc}
0 & . . & 0 \\
. & . . & . . \\
. . & . . & . . \\
0 & . . & 0
\end{array}\right)
$$

$\frac{N}{2} \times \frac{N}{2}-1$ non zero and $\frac{N}{2}-1 \times \frac{N}{2}$ zero matrices respectively and finally,

$$
\mathbf{D}_{22}=\left(\begin{array}{ccc}
-\left(1+r_{j}+C_{1 j} G_{j}\right) & 1+C_{2 j} G_{j+1} & 0 \\
r_{j+1}+C_{0(j+1)} G_{j} & -\left(1+r_{j+1}+C_{1(j+1)} G_{j+1}\right) & 1+C_{2(j+1)} G_{j+2} \\
. . & . . & . . \\
. . & . . & . \\
0 & r_{j+\frac{N}{2}-2}+C_{0\left(j+\frac{N}{2}-2\right)} G_{j+\frac{N}{2}-3} & -\left(1+r_{j+\frac{N}{2}-2}+C_{1\left(j+\frac{N}{2}-2\right)} G_{j+\frac{N}{2}-2}\right)
\end{array}\right)
$$

where $j=\frac{N}{2}+1$ and $\frac{N}{2}-1 \times \frac{N}{2}-1$ tridiagonal matrix. Let us define

$$
\begin{gathered}
\boldsymbol{\epsilon}=\left(\epsilon_{0}, \epsilon_{1}, . ., \epsilon_{\frac{N}{2}-1}, \epsilon_{\frac{N}{2}+1}, . ., \epsilon_{N-1}\right)^{t}, \\
\mathbf{T}=\left(T_{0}, T_{1}, . ., T_{\frac{N}{2}-1}, T_{\frac{N}{2}+1}, . ., T_{N-1}\right)^{t},
\end{gathered}
$$

$1 \times N-1$ matrices.

Let us assume that $C_{0 k} G_{k-1} \geq 0$ and $C_{1 k} G_{k} \geq 0$ for all $k=1,2, . ., \frac{N}{2}$ then matrices $\mathbf{D}_{11}$ and $\mathbf{D}_{22}$ are invertible. Moreover $\mathbf{D}_{11}^{-1}>0$ a positive definite and $\mathbf{D}_{22}^{-1}<0$ a negative definite. Also

$$
\left|\mathbf{D}_{12} \mathbf{D}_{22}^{-1} \mathbf{D}_{21} \mathbf{D}_{11}^{-1}\right|=0<1
$$

Therefore $\mathbf{D}$ is invertible [4].

Let us define,

$$
d_{2}^{*}=\left\|\mathbf{D}_{12} \mathbf{D}_{22}^{-1}\right\|_{\infty} \quad \text { and } \quad d_{* 1}=\left\|\mathbf{D}_{21} \mathbf{D}_{11}^{-1}\right\|_{\infty}
$$

and

$$
S_{j}^{*}= \begin{cases}C_{0 j} G_{j-1}-C_{1(j+1)} G_{j+1}+C_{2(j+2)} G_{j+2}, & 1 \leq j \leq \frac{N}{2}-2 \\ C_{0 j} G_{j-1}-C_{1(j+1)} G_{j+1}-1, & j=\frac{N}{2}-1 \\ r_{\frac{N}{2}}+C_{0 \frac{N}{2}} G_{\frac{N}{2}-1}, & j=\frac{N}{2}\end{cases}
$$

and

$$
S_{* j}= \begin{cases}-r_{j}-C_{1 j} G_{j}+C_{2 j} G_{j+1}, & j=\frac{N}{2}+1 \\ C_{0 j} G_{j-1}-C_{1 j} G_{j}+C_{2 j} G_{j+1} & \frac{N}{2}+2 \leq j \leq N-2 \\ C_{0 j} G_{j-1}-C_{1 j} G_{j}-1, & j=N-1\end{cases}
$$


sum of the elements in a row of the matrices $\mathbf{D}_{11}$ and $\mathbf{D}_{22}$ respectively. Let $S_{\min }^{*}=\min \left\{S_{j}^{*}\right\}, 1 \leq j \leq \frac{N}{2}$ and $S_{* \max }=\max \left\{S_{* j}\right\}, \frac{N}{2}+\leq j \leq N-1$. Thus

$$
d_{2}^{*}=\frac{1+C_{2 \frac{N}{2}} G_{\frac{N}{2}+1}}{\left|S_{* N-1}\right|} \text { and } d_{* 1}=0
$$

and

So, we have

$$
\left\|\mathbf{D}_{11}^{-1}\right\|_{\infty} \leq \frac{1}{S_{\min }^{*}} \quad \text { and } \quad\left\|\mathbf{D}_{22}^{-1}\right\|_{\infty} \leq \frac{1}{\left|S_{* \max }\right|}
$$

$$
\frac{1}{S_{\min }^{*}}=\max \left\{\frac{1}{S_{\min }^{*}}, \frac{1}{\left|S_{* \max }\right|}\right\}
$$

But we know that

$$
\left\|\mathbf{D}^{-1}\right\|_{\infty} \leq \frac{\max \left\{\left\|\mathbf{D}_{11}^{-1}\right\|_{\infty},\left\|\mathbf{D}_{22}^{-1}\right\|_{\infty}\right\}\left(1+d_{2}^{*}\right)\left(1+d_{* 1}\right)}{\left(1+d_{2}^{*}\right)+\left(1+d_{* 1}\right)-\left(1+d_{2}^{*}\right)\left(1+d_{* 1}\right)}
$$

Hence using (13) and (14) in above equation, we will obtain,

$$
\left\|\mathbf{D}^{-1}\right\|_{\infty} \leq \frac{1}{S_{\min }^{*}}\left(1+\frac{1+C_{2 \frac{N}{2}} G_{\frac{N}{2}+1}}{\left|S_{* N-1}\right|}\right) .
$$

Using (15) and from (11) we have,

$$
\|\boldsymbol{\epsilon}\|_{\infty} \leq \frac{1}{S_{\min }^{*}}\left(1+\frac{1+C_{2 \frac{N}{2}} G_{\frac{N}{2}+1}}{\left|S_{* N-1}\right|}\right)\|\mathbf{T}\|_{\infty} .
$$

It follows from (6) and (16) that $\|\boldsymbol{\epsilon}\| \rightarrow 0$ as $h_{i} \rightarrow 0$. Thus we conclude that method (5) converges and the order of the convergence of method (5) is at least cubic.

\section{$5 \quad$ Numerical Results}

We have considered linear and nonlinear model problems to perform the numerical experiment. In each model problem, we took constant $r_{i}=\frac{h_{i+1}}{h_{i}}$. We have used the following formula in computation of $M A E$,

$$
M A E=\max _{1 \leq i \leq N}\left|u\left(x_{i}\right)-u_{i}\right| .
$$

The order of the convergence $\left(O_{N}\right)$ of the method (5) is estimated by the formula

$$
\left(O_{N}\right)=\log _{m}\left(\frac{M A E_{N}}{M A E_{(m N)}}\right),
$$

where $\mathrm{m}$ can be estimated by considering the ratio of $N^{\prime} s$.

We have used Gauss-Seidel and Newton-Raphson method to solve the system of linear and nonlinear equations arises from equation (5). All computations were performed on a Windows 2007 Ultimate operating system in the GNU FORTRAN environment version 99 compiler (2.95 of gcc) on Intel Core i3-2330M, 2.20 Ghz PC. The solutions are computed on $N$ nodes and iteration is continued until either the maximum difference between two successive iterates is less than $10^{-8}$ or the number of iteration reached $10^{2}$.

Problem 1. The model linear problem given by

$$
u^{\prime \prime}(x)=\frac{2.0}{x^{2}} u(x)-\frac{1}{x}, \quad 1 \leq x \leq 2
$$

subject to boundary and internal conditions

$$
u\left(\frac{3}{2}\right)=\frac{-27}{152}, \quad \text { and } \quad u(2)=0 .
$$


The constructed analytical solution of the problem is $u(x)=\frac{1}{2} x-\frac{5}{38} x^{2}-\frac{18}{19 x}$. The $M A E$ for different values of $N$, $r_{i}$ and no. of iterations Iter. required to achieve $M A E$ are presented in Table 1.

Problem 2. The model nonlinear problem given by

$$
u^{\prime \prime}(x)=\frac{1.0}{2}(1.0+u(x)+x)^{3}, \quad 0 \leq x \leq 1
$$

subject to boundary and internal conditions

$$
u(1)=0, \quad \text { and } \quad u\left(\frac{1}{2}\right)=\frac{1}{3} .
$$

The constructed analytical solution of the problem is $u(x)=\frac{2.0}{2-x}-x-1.0$. The $M A E$ for different values of $N, r_{i}$ and no. of iterations Iter. required to achieve $M A E$ are presented in Table 2.

Table 1: Maximum absolute error (Problem 1).

\begin{tabular}{|c|c|cccc|}
\hline \multirow{2}{*}{$r_{i}$} & & \multicolumn{4}{|c|}{$\mathrm{N}$} \\
\cline { 3 - 7 } 1.0 & MAE & 4 & 8 & 16 & 32 \\
\cline { 2 - 6 } & Iter. & 3 & 5 & 8 & 16 \\
\hline \multirow{2}{*}{0.95} & MAY & $.44887944(-3)$ & $.46846115(-4)$ & $.68513968(-5)$ & $.25598627(-5)$ \\
\cline { 2 - 6 } & Iter. & 2 & 5 & 9 & 17 \\
\hline \multirow{2}{*}{0.9} & MAY & $.71131869(-3)$ & $.10639115(-3)$ & $.25865278(-4)$ & $.12990676(-4)$ \\
\cline { 2 - 6 } & Iter. & 3 & 5 & 9 & 16 \\
\hline \multirow{2}{*}{0.8} & MAY & $.15231339(-2)$ & $.41204377(-3)$ & $.22166653(-3)$ & $.19693060(-3)$ \\
\cline { 2 - 6 } & Iter. & 3 & 5 & 8 & 17 \\
\hline
\end{tabular}

Table 2: Maximum absolute error (Problem 2).

\begin{tabular}{|c|c|cccc|}
\hline \multirow{2}{*}{$r_{i}$} & & \multicolumn{5}{|c|}{$\mathrm{N}$} \\
\cline { 3 - 6 } & & 4 & 8 & 16 & 32 \\
\hline 1.0 & MAE & $.21792612(-3)$ & $.90324320(-5)$ & $.59839925(-6)$ & $.10335740(-6)$ \\
\cline { 2 - 6 } & Iter. & 6 & 6 & 14 & 19 \\
\hline \multirow{2}{*}{0.95} & MAY & $.99826932(-4)$ & $.32790463(-5)$ & $.16452258(-5)$ & $.58915737(-6)$ \\
\cline { 2 - 6 } & Iter. & 2 & 7 & 11 & 15 \\
\hline \multirow{2}{*}{0.9} & MAY & $.79323072(-4)$ & $.31364336(-4)$ & $.95603837(-5)$ & $.37932873(-5)$ \\
\cline { 2 - 6 } & Iter. & 4 & 6 & 10 & 19 \\
\hline \multirow{2}{*}{0.8} & MAY & $.72076422(-3)$ & $.19227999(-3)$ & $.91814261(-4)$ & $.85381209(-4)$ \\
\cline { 2 - 6 } & Iter. & 5 & 6 & 9 & 19 \\
\hline
\end{tabular}

We have described a method for numerical solution of two point boundary value problems with nonuniform mesh and internal boundary condition. Linear and nonlinear model problems considered to illustrate the computing performance of the proposed method. Numerical results for example 1 which is presented in table 1 , for different values of $N$ and $r_{i}$. We observed from the computational results that the decrease in $h_{i}$, decrease in $M A E$ for same $r_{i}$. The order of the convergence reduced substantially as we decreases the value of $r_{i}$ from 1 to less than 1 . Thus we conclude that the order of the method depends on choice the $r_{i}$. Similar observation can be found in result of example 2. Over all method (5) is convergent. 


\section{Conclusion}

A finite difference method to find the numerical solution of two point boundary value problems with nonuniform mesh and internal condition has been developed. This method is a Numerov type method and transforms the problem (1) into system of algebraic equations given by (5). The propose method produces good approximate numerical value of the solution of the model problems with nonuniform step size. The numerical results for the model problems showed that the proposed method is computationally efficient. The rate of convergence of the present method depends on $r_{i}$, the ratio of step sizes and the value of $r_{i}$ very close to 1 is cubic. The idea presented in this article leads to the possibility to develop finite difference methods for the numerical solution of higher order boundary value problems with internal conditions. Works in these directions are in progress.

\section{References}

[1] U.Ascher, R. M. M. Mattheij, and R. D. Russell, Numerical Solution of Boundary Value Problems for Ordinary Differential Equations. Prentice-Hall, Englewood Cliffs, NJ (1988).

[2] J. V. Baxley, Nonlinear Two Point Boundary Value Problems in Ordinary and Partial Differential Equations (Everrit, W.N. and Sleeman , B.D. Eds.). 46-54, Springer-Verlag, New York (1981).

[3] A.Friedman, Variational principles and free-boundary problems, Wiley (1982) (reprinted Krieger, Malabar, FL, 1988).

[4] M. I. Gil, Invertibility Conditions for Block Matrices and Estimates for Norms of Inverse Matrices. Rocky Mountain Journal of Mathematics Vol.33, Num.4,1323-1335 (2003).

[5] H.B. Keller, Numerical Methods for Two Point Boundary Value Problems. Waltham, Mass. Blaisdell Publ. Co. (1968).

[6] N.Kikuchi, and J.T.Oden, Contact problems in Elasticity. SIAM Publishing Company, Philadelphia (1998).

[7] J. D.Lambert, Numerical Methods for Ordinary Differential Systems . John Wiley, England (1991).

[8] W. H.Press, S. A.Teukolsky, W. T. Vetterling and B. P.Flannery, NUMERICAL RECIPES-The Art of Scientific Computing. Cambridge University Press, Cambridge, New York, Melbourne, Madrid, Cape Town, Singapore, So Paulo (2007). 